\title{
Role of platelet function and platelet membrane glycoproteins in children with primary immune thrombocytopenia
}

\author{
WEN-JUN LIU, JING BAI, QU-LIAN GUO, ZHE HUANG, HONG YANG and YONG-QI BAI \\ Department of Pediatrics, The Affiliated Hospital of Southwest Medical University, Luzhou, Sichuan 646000, P.R. China
}

Received February 15, 2016; Accepted July 11, 2016

DOI: $10.3892 / \mathrm{mmr} .2016 .5504$

\begin{abstract}
The aim of the present study was to examine and understand changes in platelet functions prior to and after the treatment of primary immune thrombocytopenia (ITP) in children. An automatic hematology analyzer and whole blood flow cytometry were used to detect immature platelet fraction (IPF), IPC and membrane glycoproteins (CD62p, PAC-1 and CD42b) in ITP children (ITP group), children with complete response after ITP treatment (ITP-CR group) and children with elective surgery (normal control group). The results showed that, levels of platelet count (PLT) and plateletcrit in the ITP group were lower alhtough the levels of mean platelet volume, platelet distribution width and platelet-large cell ratio (P-LCR) were higher than those in the normal control and ITP-CR groups. PLT in the ITP-CR group was lower than that in the normal controls. Additionally, IPF\% was higher in the normal control and ITP-CR groups, IPC was lower in the ITP group compared to the normal control and ITP-CR groups. Furthermore, prior to ADP activation, the expression levels of CD62p, PAC-1 and CD42b in the ITP group were lower in ITP group than those in the normal control and ITP-CR groups. The expression level of PAC-1 was lower in the ITP-CR and normal control groups. No differences were identified in CD62p and CD42b expression levels. Following ATP activation, CD62p, PAC-1 and CD42b expression in the ITP group was lower than that in the normal control and ITP-CR groups. PAC-1 expression was lower while CD62p expression was higher in the ITP-CR group compared to the normal control group. In conclusion, the activation of platelets in ITP children was low. Decreased platelet function, platelet parameters and platelet glycoproteins may be
\end{abstract}

Correspondence to: Dr Wen-Jun Liu, Department of Pediatrics, The Affiliated Hospital of Southwest Medical University, 25 Taiping Street, Luzhou, Sichuan 646000, P.R. China

E-mail: 1wjlyfy@qq.com

Key words: primary immune thrombocytopenia, platelet parameters, platelet function used as markers for monitoring the treatment efficacy in ITP children.

\section{Introduction}

Primary immune thrombocytopenia (ITP) is a common acquired autoimmune hemorrhagic disorder, accounting for $30 \%$ of the total hemorrhagic disorders (1). The annual incidence of ITP in children was approximately 1.9-6.4/100,000 (2). The clinical manifestations of ITP were predominantly spontaneous skin and mucosal bleeding, while the complication of intracranial hemorrhage occurred in severe patients, leading to death. The majority of children presented acute course, while approximately $20 \%$ children were transformed into chronic ITP (3).

The main mechanism of pathogenesis of this disease was increased platelet destruction and insufficient thrombocytopoiesis due to abnormal cellular and humoral immunity $(4,5)$. The complicated pathogenesis mechanism of ITP was recently identified (6). Investigators found apoptosis of platelets in ITP children, and speculated that platelet apoptosis was also a pathogenesis mechanism of ITP $(7,8)$. Currently, there is no uniform 'gold standard' mechanism of pathogenesis. Clinical diagnosis is mainly an exclusive procedure and is dependent on bone marrow aspiration, which is traumatic and not routinely recommended. Previous findings $(9,10)$ showed that changes of the platelet-associated parameters [mean platelet volume (MPV), platelet distribution width (PDW), platelet-large cell ratio (P-LCR), immature platelet fraction (IPF)\%] were used to diagnose thrombocytopenia due to various causes, and used as indicators of relapse and efficacy. Long-term clinical diagnosis and treatment also indicated that the hemorrhagic symptoms of some children were not proportional to the reduction of the platelet count (PLT), which indicated that hemorrhage in ITP children was asssociated with the reduction of platelet count, as well as abnormal platelet function. Therefore, only platelet count is not sufficient to evaluate disease severity in children, and monitoring of individual platelet function should also be considered. Previously employed methods for the detection of platelet function were limiting and were not optimal to assess the many characteristics of platelet. Therefore, the development of a rapid, sensitive and convenient detection method is important to assess this disease, and understand platelet function. 
The development of flow cytometry (FCM) and various monoclonal antibodies that could be labeled with fluorescein, led to several tools for the detection of platelet function becoming available.

Whole blood FCM was used to measure the percentage or fluorescence intensity of fluorescence-labelled platelet-specific membrane glycoproteins and activation markers, in order to assess the activation of circulating platelets and the in vitro response to activators by platelets, and evaluate platelet function. This technique simplified the treatment procedure of samples, avoided the artificial activation of platelet in vitro and prevented the loss of platelet subset. In addition, the blood sample volume was small, thus this technique was especially useful in the evaluation of the platelet function in the children with thrombocytopenia. Regarding relevant antibodies, Chen et al (11) compared the monoclonal antibody-specific immobilization of platelet antigens (MAIPA) and FCM in the detection of the sensitivity and specificity of GPIIb/IIIa and GPIIb/IIIa antibodies, and identified no significant differences. However, the sensitivity of FCM was higher than that of MAIPA. This observation indicated that FCM could be used as a new clinical diagnostic method (12-14).

Alterations in platelet function in ITP patients is controversial. Wang et al (15) demonstrated decreased activation and lower function of platelet in vitro and in vivo in ITP patients. Psaila et al (16) compared the expression of membrane glycoproteins in the platelets in ITP patients and MDS patients, and found high activation and in vitro response of the platelet in ITP patients. Therefore, further investigation of the platelet function change was required. The abovementioned study detected activation markers of GPIIb/IIIa on the platelet surface, such as PAC-1, granule membrane glycoprotein (CD62p), CD42b and IPF, evaluated the function of peripheral platelet and the change of new platelet in ITP patients prior to and after treatment, and assessed the change of platelet function in ITP patients after initial diagnosis and treatment.

The aim of the present study was to detect PAC-1, CD62p, CD $42 b$ and IPF by trace whole blood FCM, evaluate the function of peripheral platelet and the change of new platelet in ITP patients before and after treatment, assess the change of platelet function in ITP patients after initial diagnosis and treatment, analyze the change of platelet-associated parameters, such as platelet count (PLT), MPV, plateletcrit (PCT), PDW and platelet-large cell ratio, and provide the basis of the diagnosis, and the interpretation of disease course and efficacy.

\section{Materials and methods}

Materials. Fluorescein-labeled anti-platelet monoclonal antibodies used were: fluorescein isothiocyanate (FITC)labeled anti-platelet GPIIb/IIIa monoclonal mouse antibody (PAC-1-FITC; cat. no. 340507); phycoerythrin (PE)-labeled anti-platelet GMP-140 mouse monoclonal antibody (CD62p-PE; cat. no. 348107); peridinin chlorophyll protein-labeled GPIIIa monoclonal mouse antibody (CD61PercP; cat. no. 340506); PE-labeled mouse IgG (MIgG-PE; cat. no. 349043); phycocyanin-labeled anti-platelet GPIb-IX monoclonal mouse antibody (CD42b-APC; cat. no. 551061); and phycocyanin-labeled mouse IgG (MIgG-APC; cat. no. 555751). Thiazole orange (TO) $(1.0 \mu \mathrm{g} / \mathrm{ml}$; Sigma,
St. Louis, MO, USA); RGDS (Arg-Gly-Asp-Ser) peptide as a blocker of PAC-1, and synthetic arginyl-glycyl-Asp-serine peptide, (Sigma) were also used. Other monoclonal antibodies were purchased from Becton-Dickinson (Franklin Lakes, NJ, USA). The following were also used: $0.2 \mathrm{~mol} / \mathrm{l}$ ADP (Sigma); $1 \%$ paraformaldehyde solution; phosphate-buffered saline (PBS); FCM, a BD FACSCanto ${ }^{\mathrm{TM}}$ II high-speed sorter manufactured by Becton-Dickinson; and an automatic hematology analyzer, BC-6800, Mindray (Shenzhen, China).

Subjects. All the subjects were inpatients at the Affiliated Hospital of Luzhou Medical College (Sichuan, China) between March 2014 and February 2015. All the patients agreed to participate in the current study and signed written informed consent. The patients were divided into three groups: i) Normal control: 17 children prior to elective surgery at the Department of Pediatric Surgery, including 12 male and 5 female patients, with a mean age of $4.05 \pm 1.89$ years. The children had inguinal hernia, with a mean platelet count of $309.2 \pm 54.06 \times 10^{9} / 1$. None of the children had a previous history of circulatory diseases, immunological diseases, malignancy or transfusion. None of the children had any infection signs 1 week prior to hospitalization, and had no drugs 1 week prior to blood sampling. The children had normal complete blood count and blood samples were collected prior to surgery; ii) primary ITP: all the children met the diagnostic criteria of ITP (17), including 12 male and 6 female patients. The mean age of $3.8 \pm 1.4$ years, the mean onset time (from the onset of disease to presentation in hospital) was 1-6 days and the mean platelet count was $28.61 \pm 10.42 \times 10^{9} / 1$. No children had drugs that could affect platelet function 3 months prior to admission, such as corticosteroids, immunosuppressants, heparin, or any relevant treatment. This disease was not accompanied by significant infectious symptoms, and required no other anti-infection drugs. ITP was confirmed by the morphological analysis of bone marrow cells, which were collected prior to drug administration; and iii) complete response to primary ITP (ITP-CR): the children met the efficacy criteria (18) with a mean platelet count of $236.9 \pm 40.9 \times 10^{9} / 1$. The differences in the age and gender between the treatment and control groups were not statistically significant $(\mathrm{p}>0.05)$.

Approval of the study was obtained from the Ethics Committee of the Affiliated Hospital of Luzhou Medical College.

Detection methods. Platelet membrane glycoproteins, such as PAC-1, CD62p and CD42b, were measured. Venous blood ( $3 \mathrm{ml}$ ) was collected and the middle section of $1.8 \mathrm{ml}$ whole blood was added into an anticoagulation tube containing sodium citrate, mixed gently and processed for analysis. Platelet activation was carried out by adding $50 \mu \mathrm{l}$ ADP into a Falcon tube (Becton-Dickinson Company, Franklin Lakes, NJ, USA) followed by $450 \mu 1$ blood with anticoagulant, mixed gently by vortexing and incubated at $37^{\circ} \mathrm{C}$ for $10 \mathrm{~min}$. Expression of PAC-1, CD62p and CD42b was measured by three-color fluorescence analysis with FCM. In separate tubes, $20 \mu 1$ of PE-isotype control antibody, CD61-PercP, PAC-1-FITC and MIgG-APC, and $10 \mu \mathrm{l}$ RGDS or $20 \mu \mathrm{l}$ CD62p-PE, CD61-PercP, PAC-1-FITC and CD42b-APC were 
A
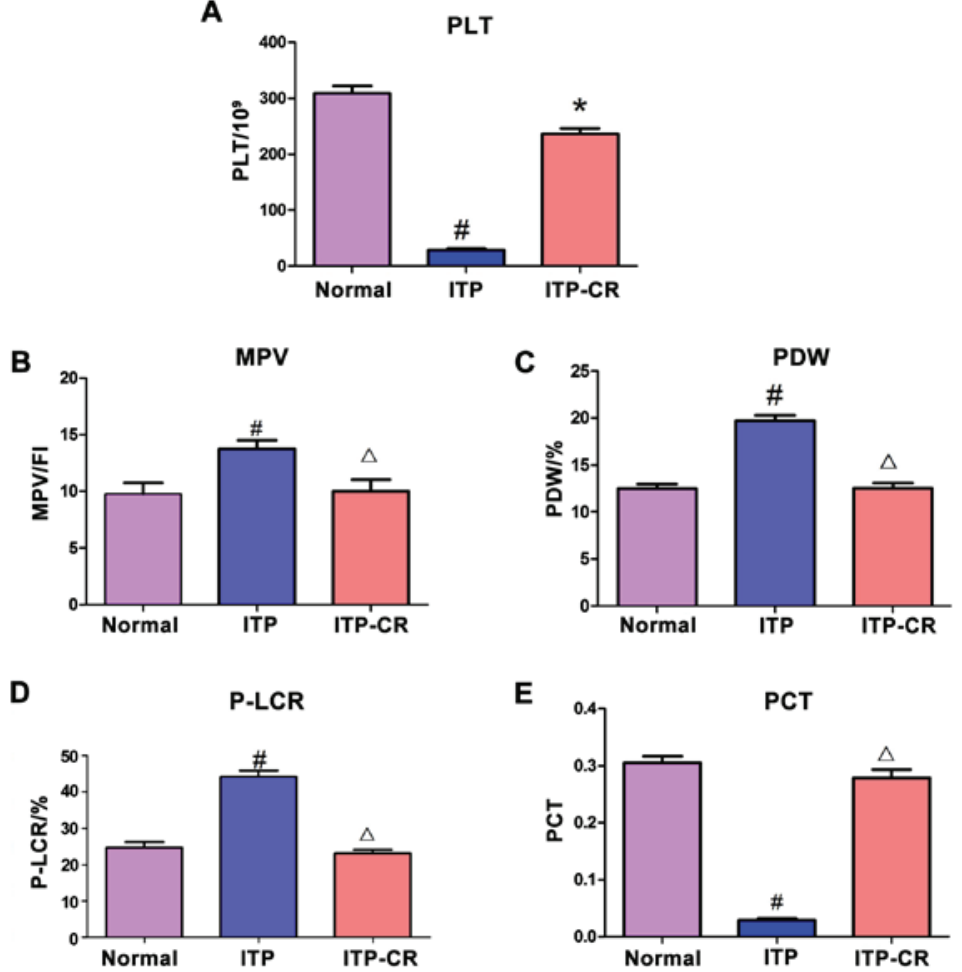

Figure 1. Comparison of platelet parameters. (A) The expression of PLT in 3 groups, (B) the expression of MPV in 3 groups, (C) the expression of PDW in 3 groups, (D) the expression of P-LCR in 3 groups, and (E) the expression of PCT in 3 groups. ${ }^{~} \mathrm{P}<0.05$, ITP-CR vs. normal control group; ${ }^{*} \mathrm{P}<0.05$, in comparison to the normal control group; ${ }^{\triangle} \mathrm{P}>0.05$, in comparison to the normal control group. PLT, platelet count; MPV, mean platelet volume; PDW, platelet distribution width; P-LCR, platelet-large cell ratio; PCT, plateletcrit.

A

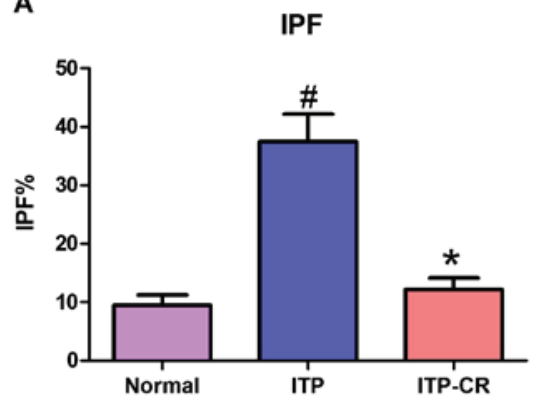

B

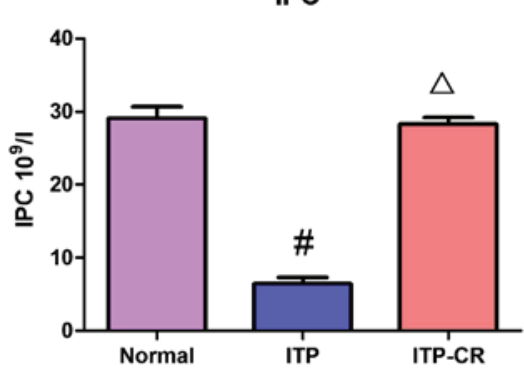

Figure 2. Expression of IPF\% and IPC. (A) Expression of IPF\% in 3 groups, and (B) expression of IPC in 3 groups. ${ }^{\#} \mathrm{P}<0.05$, ITP-CR vs. normal control group; ${ }^{*} \mathrm{P}<0.05$, in comparison to the normal control group; ${ }^{\wedge} \mathrm{p}>0.05$, in comparison to the normal control group. IPF, immature platelet fraction.

added. Then, $5 \mu 1$ non-activated and activated whole blood was added into the tubes of the treatment groups, and $5 \mu 1$ non-activated whole blood was added into the control tube, mixed gently, and incubated at room temperature $\left(25^{\circ} \mathrm{C}\right)$ in the dark for $20 \mathrm{~min}$. Subsequently, $1 \%$ paraformaldehyde was added $\left(2-8^{\circ} \mathrm{C}, 1 \mathrm{ml}\right)$ into each tube, mixed completely, and placed at $2-8^{\circ} \mathrm{C}$ in the dark for $30 \mathrm{~min}$. Using FCM, signal for 10,000 platelets was acquired, and the positive expression rate of CD62p, PAC-1 and CD42b was calculated (19).

Platelet-associated parameters (PLT, MPV, PDW, PCT and P-LCR) were measured. Venous blood (4-ml) was collected into an anticoagulant tube containing EDTA with a 5-ml syringe, 2-ml whole blood was reserved, and the remaining blood was used in the measurement of platelet-associated parameters by an automatic hematology analyzer. Whole blood (5- $\mu \mathrm{l})$ was placed into the treatment tubes, followed by $5 \mu \mathrm{l}$ CD42b-APC and $50 \mu 1$ TO. Whole blood (5- $\mu \mathrm{l})$ was placed into the control tube, followed by $5 \mu 1 \mathrm{CD} 42 \mathrm{~b}-\mathrm{APC}$ and $50 \mu \mathrm{l}$ PBS. The tubes were mixed gently, incubated at room temperature in the dark for $15 \mathrm{~min}$; and then, $1 \%$ paraformaldehyde was added $\left(2-8^{\circ} \mathrm{C}, 1 \mu \mathrm{l}\right)$ into each tube, and mixed completely. The samples were analyzed within $45 \mathrm{~min}$. Signals for 10,000 platelets were obtained, IPC was measured and IPF\% was calculated.

Statistical analysis. Experimental data were presented as mean \pm SD and analyzed using SPSS 11.5 software (SPSS Inc., Chicago, IL, USA). The Student's t-test or rank-sum test was used for comparisons between the two treatment groups. The one-sample t-test or rank-sum test was used for the comparison between the treatment and control groups. $\mathrm{P} \leq 0.05$ was considered to indicate a statistically significant difference. 
A

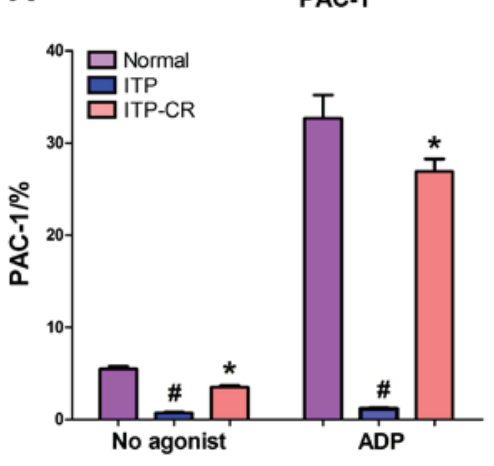

B
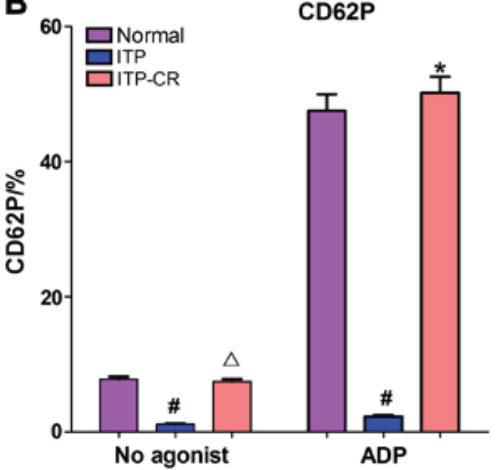

CD42b

C

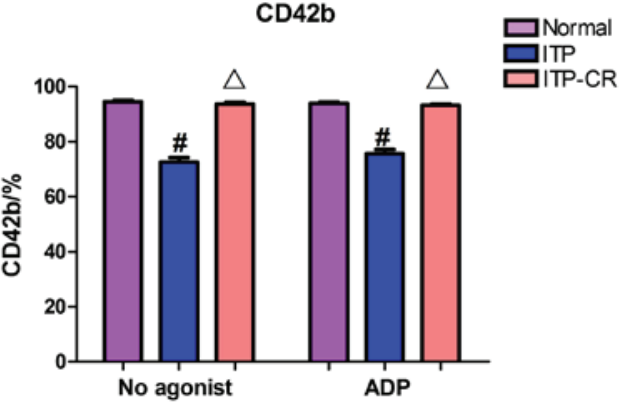

Figure 3. Expression of (A) PAC-1, (B) CD62p and (C) CD42b. " $\mathrm{P}<0.05$, ITP-CR group vs. normal control group; ${ }^{\mathrm{p}}<<0.05$, in comparison to normal control group; and ${ }^{\wedge} \mathrm{p}>0.05$, in comparison to normal control group.

A

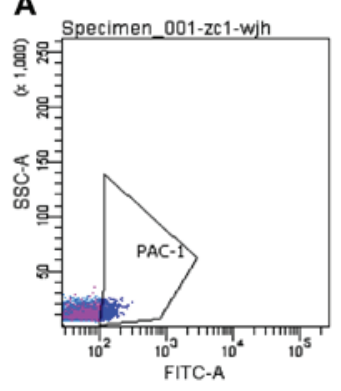

B

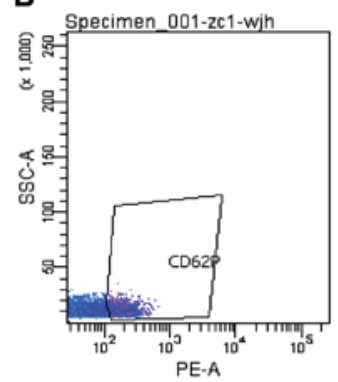

C

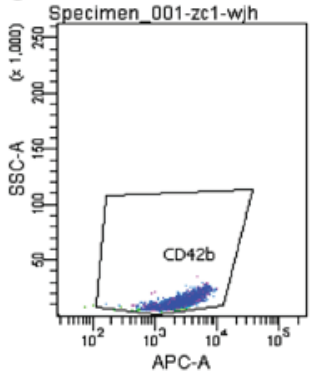

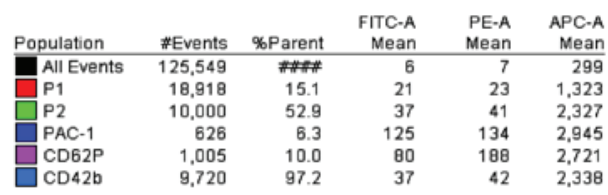

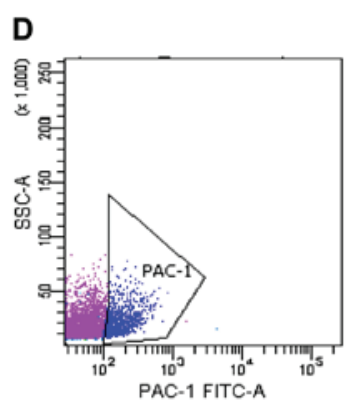

E

$\mathbf{F}$
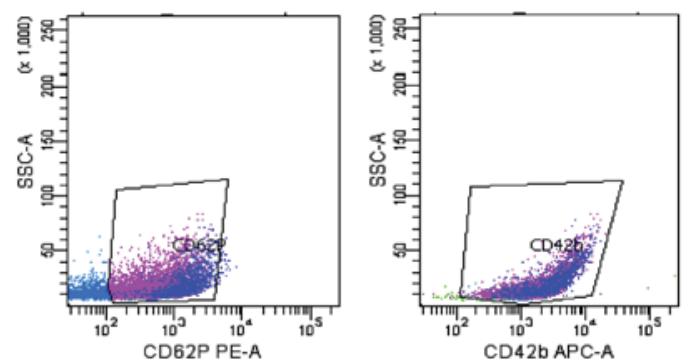

CD42b APC-A

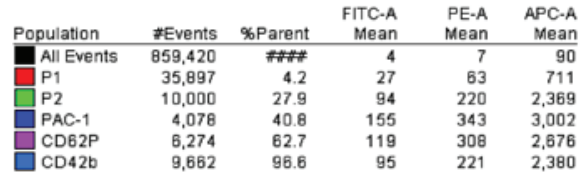

Figure 4. Expression of PAC-1, CD62p and CD42b in the normal control group before and after platelet activation by ADP. P2 indicated 10,000 platelets obtained. The polygon in panels (A-C) is the expression of PAC-1, CD62p and CD42b before platelet activation by ADP in the normal control group. The polygon in panels (D-F) shows the expression of PAC-1, CD62p and CD42b after platelet activation by ADP in the normal control group. 
A

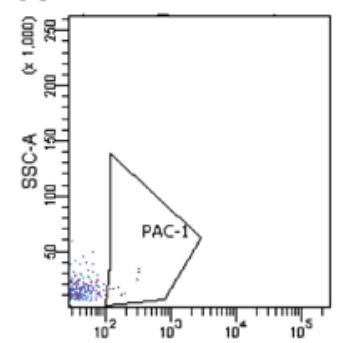

B

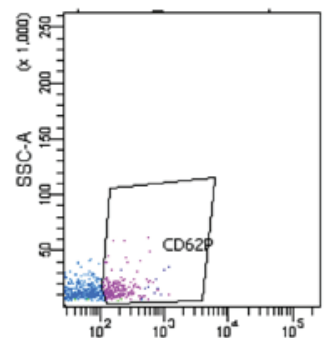

C

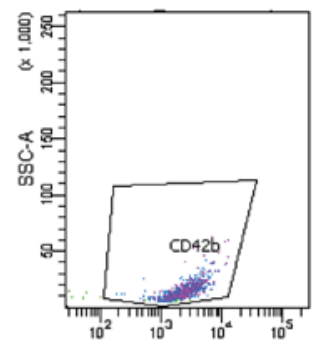

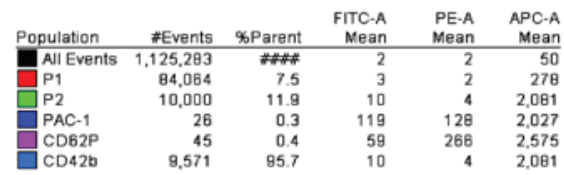

D

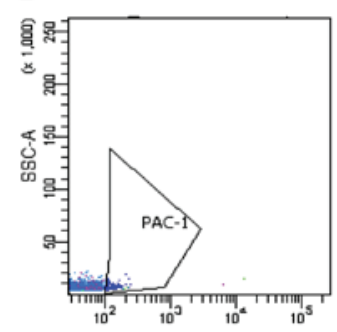

E

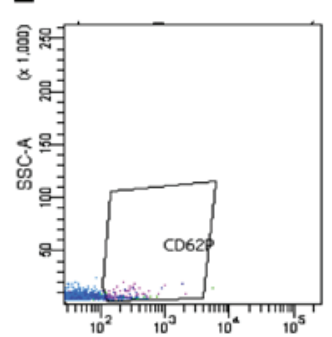

$\mathbf{F}$
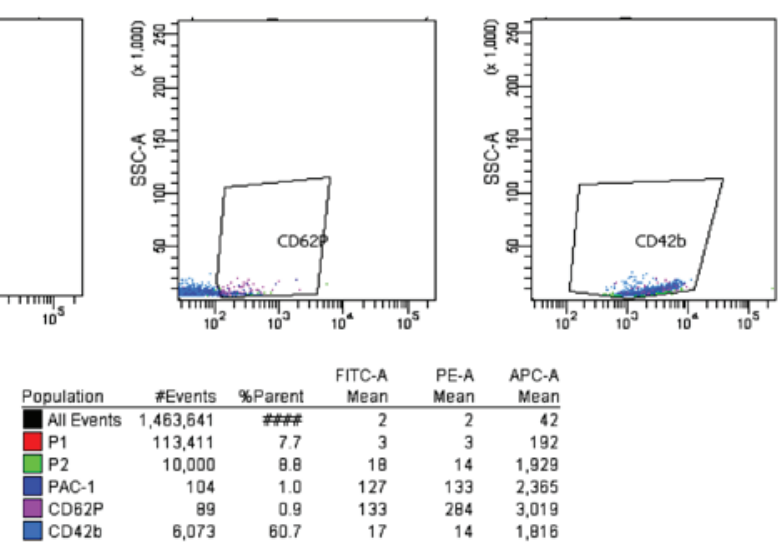

Figure 5. Expression of PAC-1, CD62p and CD42b in the ITP group before and after platelet activation by ADP (FCM). P2 indicated 10,000 platelet obtained. The polygon in panels (A-C) shows the expression of PAC-1, CD62p and CD42b before platelet activation by ADP in the normal control group. The polygon in panels (D-F) shows the expression of PAC-1, CD62p and CD42b after platelet activation by ADP in the normal control group. FCM, flow cytometry; ITP immune thrombocytopenia.

\section{Results}

Measurement of platelet parameters. As shown in Fig. 1, PLT and PCT in the ITP group had a lower expression level compared to those in the normal control and ITP-CR groups $(\mathrm{p}<0.05)$, while the MPV, PDW and P-LCR in ITP group were higher than those in the normal control and ITP-CR groups $(p<0.05)$. Differences in the expression of MPV, PDW, PCT and P-LCR between the ITP-CR and normal control groups were not statistically significant ( $p>0.05$ ), whereas PLT was lower in the ITP-CR group $(\mathrm{p}<0.05)$.

Measurement of IPF\% and IPC. As shown in Figs. 2 and 7, IPF\% in the ITP group was higher than that in the ITP-CR and normal control groups $(\mathrm{p}<0.05)$. IPC in the ITP group was lower than that in the ITP-CR and normal control groups $(p>0.05)$. IPF\% was higher $(p<0.05)$ and IPC was lower in ITP-CR group in comparison to the normal control group. The differences were not statistically significant.

Measurement of platelet membrane glycoproteins prior to and after platelet activation by ADP. As shown in Figs. 3-6, prior to platelet activation by ADP, the expression levels of CD62p, PAC-1 and CD42b in the ITP group were lower than those in ITP-CR and normal control groups $(\mathrm{p}<0.05)$. PAC-1 was lower in the ITP-CR compared to that in the normal control group ( $\mathrm{p}<0.05)$. Differences in CD62p and CD42b were not statistically significant $(\mathrm{p}>0.05)$. After platelet activation by ADP, the expression levels of CD62p, PAC-1 and CD42b in the ITP group were lower than those in the ITP-CR and normal control groups $(p<0.05)$. PAC-1 was lower and CD62p was higher in the ITP-CR group than that in the normal control group ( $\mathrm{p}<0.05)$. Differences in CD42b were not statistically significant $(\mathrm{p}>0.05)$.

\section{Discussion}

Platelet is the smallest cell in whole blood cells, originating from mature megakaryocytes cytoplasm. It has the functions of adhesion, accumulation and release, and plays an important role in hemostasis (16). Platelet membrane glycoprotein (GP) is a main component of platelet membrane proteins, and plays major roles in the activation of platelet. Platelet GPs can be classified into plasma membrane and granule membrane glycoproteins based on the distribution location (19). Plasma membrane glycoproteins are predominantly located on the cell membrane of resting platelets, major GPs including the GPIb (CD42b)-IX-V complex and GPIIb/IIIa complex. 

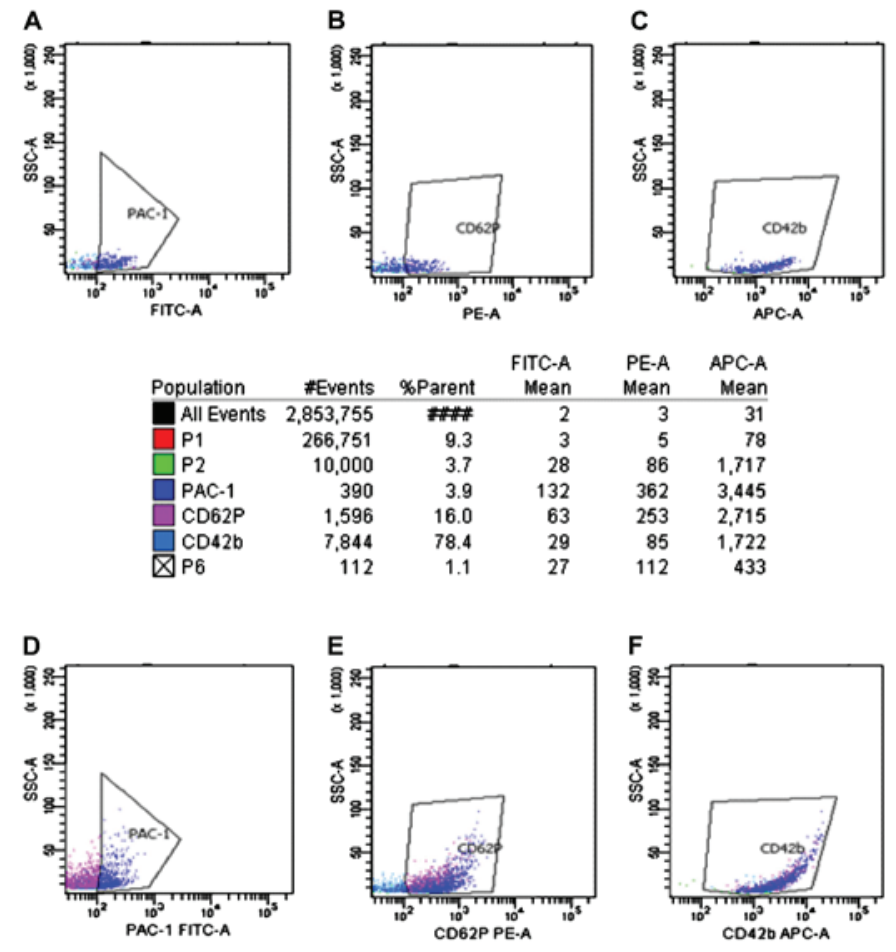

\begin{tabular}{lrrrrr} 
Population & \#Events & \%Parent & $\begin{array}{r}\text { FITC-A } \\
\text { Mean }\end{array}$ & $\begin{array}{r}\text { PE-A } \\
\text { Mean }\end{array}$ & $\begin{array}{r}\text { APC-A } \\
\text { Mean }\end{array}$ \\
\hline All Events & $1,493,736$ & 1 & 2 & 2 & 36 \\
P1 & $\mathbf{4 8 , 6 7 8}$ & 3.3 & 11 & 19 & 381 \\
\hline P2 & 10,000 & 20.5 & 45 & 88 & 1,808 \\
PAC-1 & 1,360 & 13.6 & 139 & 239 & 2,198 \\
CD62P & 6,208 & 62.1 & 168 & 313 & 2,822 \\
CD42b & 9,522 & 95.2 & 132 & 225 & 2,566 \\
$\square$ P6 & 13 & 0.1 & 91 & 91 & 360
\end{tabular}

Figure 6. The expression of PAC-1, CD62p and CD42b in the ITP group before and after platelet activation by ADP (FCM). P2 indicated 10,000 platelet obtained. The polygon in panels (A-C) represented the expression of PAC-1, CD62p and CD42b before platelet activation by ADP in normal control group. The polygon in panels (D-F) represented the expression of PAC-1, CD62p and CD42b after platelet activation by ADP in normal control group. ITP, immune thrombocytopenia; FCM, flow cytommetry.

A

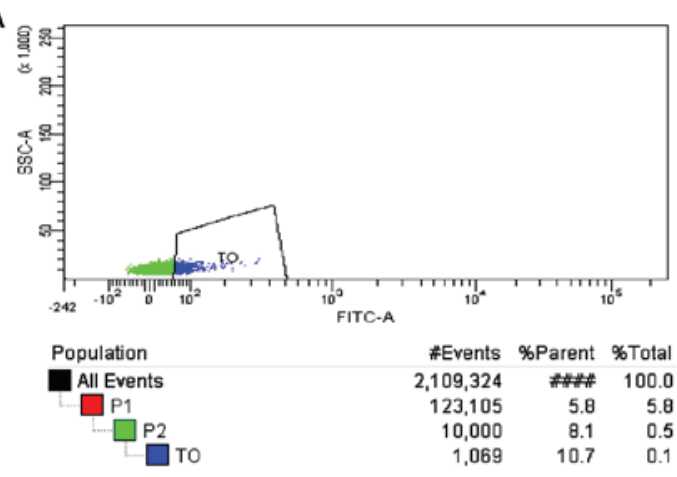

B

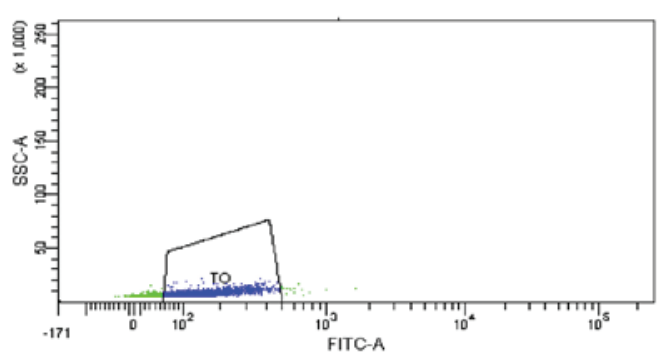

C

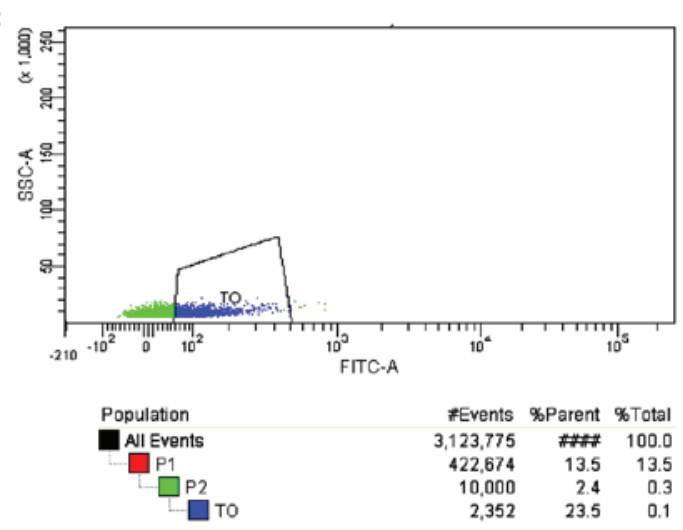

Figure 7. Expression of IPF\% in different stages of the ITP and normal control groups. TO represented reticulated platelets. (A) The polygon is the IPF\% in the normal control group; (B) the polygon is the IPF\% in the ITP group; (C) the polygon represented IPF\% in the ITP-CR group. IPF, immature platelet fraction; ITP, immune thrombocytopenia; TO, thiazole orange. 
CD42b (GPIb) is an important platelet adhesion receptor that promotes platelet to secret platelet-derived growth factors and 5-HT, accelerating the deformation and aggregation of platelets. GpIIb/IIIa complex is the most abundant membrane glycoprotein and can mediate the binding of platelet and fibrinogen $(\mathrm{Fg})$, which is the final pathway of platelet aggregation (20). PAC-1 is the monoclonal antibody in the exposed Fg-binding site after the activation of GpIIb/IIIa, and is the specific marker of the activation of GPIIb/IIIa complex and the early marker of platelet activation (21). Granule membrane glycoproteins comprise CD62p and CD63p, with CD62p also known as P-selectin. Since the increase of CD62p is not altered with time prolongation, it is considered the 'gold standard' of platelet activation markers (22). Therefore, we investigated the change of platelet function by detecting the expression of PAC-1, CD62p and CD42b (23).

Hemorrhage due to decreased platelets caused by disrupted platelet autoimmunity is currently considered as the pathogenesis mechanism of ITP (7). However, platelet function was less investigated. The present study measured the expression percentage and fluorescence intensity of three membrane glycoproteins, i.e., PAC-1, CD62p and CD42b with FCM to analyze the in vitro and in vivo activation of platelets in ITP patients. The percentage of membrane glycoproteins indicated the total amount of activated platelets, and the mean fluorescence intensity (MFI) indicated the expression of membrane glycoproteins in single-activated platelets. These factors provide an understanding of the activation capability of individual platelet. In the current study, the expression levels of PAC-1, CD62p and CD42b in ITP children were significantly lower those in the normal control group prior to and after platelet activation by ADP. This result was consistent with that by Liu and Qian (6), which demonstrated low platelet activation in vitro and in vivo in ITP children. Other studies reported that some autoantibodies may affect platelet function (24-26). As autoantibodies may bind to the antigenic epitopes on GPIIb/IIIa and GPIb (CD42b), prevent the binding of added fluorescent $\mathrm{mAb}$, the expression levels of PAC-1 and GPIb (CD42b) were decreased. Furthermore, inhibition of the initial activation steps prevented the exposure of intracellular granule membrane glycoproteins on the surface of plasma membrane, thus decreasing the expression level of CD62p. The present study also demonstrated normal MFI of CD62p in vivo and lower MFI of CD62p after platelet activation in vitro. This result demonstrated low in vivo activation of platelets in ITP children. Previous findings have shown that the inhibition or dysfunction of megakaryocytes in ITP children may cause abnormal membrane glycoproteins in new platelets in a qualitative and quantitative manner (27).

Platelet activation from the internal to the external compartment is a series of complicated physiological process, in which any section affects normal platelet activation. Further studies are required to determine which section may cause low platelet activation in ITP children. Our study also demonstrated that the expression levels of three glycoproteins gradually increased with the recovery of patients, although the expression level of PAC-1 in the recovery phase was lower than that in the normal group. This result may be related to the fact that platelet activation was enhanced and some antibodies were present due to the release of new platelets in the recovery phase in ITP children.
The expression level of CD42b in the recovery phase was similar to that in the normal control group, which may be due to less membrane glycoprotein GPIb (CD42b) in ITP patients. The percentage and MFI of platelet membrane glycoprotein CD62p were higher than those in normal children after activation by ADP. This result was consistent with Bhoria et al (28), who demonstrated enhanced platelet function in the ITP children who were in the recovery phase.

Previous studies have demonstrated enhanced platelet function in ITP children. Wang et al (29) found the positive expression rate of PAC-1 in ITP patients was significantly higher than that in healthy and non-ITP patients. Psaila et al (30) observed high intrinsic platelet activity and intrinsic platelet reactivity due to high IPF, increased GPIb on the membrane surface of circulating platelets and increased expression of activated GPIIb/IIIa and GPIb. The finding was inconsistent with our results due to: i) ITP was a group of heterogeneous diseases with complicated etiology and pathogenesis mechanisms. Activation of the individual platelet with different pathogenesis mechanism may be different. ii) The stage of disease in the selected subject may be different, as some patients may have experienced in vitro activation (31); and iii) artificial agitation in the blood sample collection, the set-up of the instruments and sample treatment, and the time of sample treatment may have affected the expression of membrane glycoproteins and the final result. A limitation of the study is that, the samples collected were limited. Thus, a larger sample size may be required in future studies.

Platelet parameter was a simple non-invasive examination, that was capable of reflecting the proliferation kinetics of platelets in vivo (32), and was significant for the early diagnosis, risk assessment and efficacy interpretation of disease. Platelet parameters included PLT, P-LCR, MPV, PCT and PDW. PLT was a common indicator for the assessment of treatment protocol and disease severity. PLT in the ITP children was significantly lower than that in the normal control group at first visit due to the disruption of platelets by autoimmunity. MPV reflected the size and metabolism of platelets and the proliferation capability of macrophage in bone marrow $(33,34)$. It was therefore useful in the assessment of platelet function and the reason for thrombocytopenia. Balduini and Noris (35) identified that the specificity of MPV in the differentiation of ITP and congenital thrombocytopenia was up to $91 \%$, and was dependent on the precision of the instrument. Korkmaz et al (9) demonstrated MPV in a relapsed patient was significantly higher than that in the patient with a primary disease, and suggested that MPV could be used to predict the relapse of ITP, while PDW was a parameter that reflected the difference of platelet volume and was positively associated with MPV when marrow proliferation was normal. Ntaios et al (10) compared the thrombocytopenia caused by ITP with that by myelosuppression, and found MPV and PDW were higher when the disruption of peripheral platelets was increased. PDW and MPV were beneficial to the diagnosis of ITP. P-LCR refers to the percentage of blood large platelets (volume of $>20 \mathrm{fl}$ ). An increase in P-LCR indicates an elevation in neonatal platelet count, and although the total number of platelets remained unchanged, it indirectly led to an increase in platelet destruction (34). PCT was the product of PLT and MPV, and the change 
of PCT was consistent with the change of PLT. The present findings demonstrated that PLT and PCR in the ITP patients were significant lower, whereas PDW, MPV and P-LCR in the ITP patients were significantly higher. Following treatment, PLT and PCR gradually increased, whereas PDW, MPV and P-LCR gradually decreased. PLT was negatively related to MPV, while MPV was positively related to PDW and P-LCR. These results were consistent with those of Fan and Wei (36), which suggested that the change of platelet parameters was beneficial to the diagnosis and efficacy interpretation of ITP patients. However, some factors may affect the detection of platelet parameters, including the time of sample storage, the method for blood collection, anticoagulant EDTA and enlarged platelet volume due to the binding of platelet to granulocytes or monocytes.

Reticulated platelets (RPs) are immature platelets that enter the blood from bone marrow, and are in the naïve phase of the process from megakaryocyte to platelet. RPs are an important indicator in assessing the ability of bone marrow to produce platelets. Various indicators can be used to identify thrombocytopenia (37-40), and be used to predict the recovery of platelets after hematopoietic stem cell transplantation (41). Liu et al (42) suggested that IPF\% was superior to PA IgG in the diagnosis of ITP. It was reported that IPF was associated with the response to ITP treatment (43). The current results have demonstrated that the IPF\% in ITP children prior to treatment was significantly increased in comparison to the normal control group. IPF\% decreased with the relief of disease following treatment. The results were consistent with those by Adly et al (44), which suggested that the disruption of peripheral platelets in ITP children was increased, and although the bone marrow hyperplasia was normal, the compensatory hyperplasia of megakaryocytes caused the release of a large number of new platelets, leading to increased IPF\% and subsequent increased PLT. As a result, the stimulation to megakaryocytes was weak and IPF\% gradually decreased. IPC was the product of PLT and IPF\%, and represented the absolute value of reticulated platelets in unit volume of peripheral blood. The present findings demonstrated that IPC prior to treatment in the ITP children was significantly decreased in comparison to the normal control group, and the response period after ITP treatment was significantly longer than that prior to treatment. IPC was in inverse proportion to IPF\%. This may be associated with the short life-span of new platelets or the disruption of new platelets following release into the blood. Barsam et al (45) suggested that IPC reflected the balance between the compensatory production of platelets by megakaryocytes in ITP children and the disruption rate of peripheral platelets, and could be used as the efficacy indicator following treatment of thrombopoietin in chronic ITP patients. Greene et al (46) suggested that IPC could be used to assess the hemorrhage risk in ITP patients better than platelet parameters, such as PLT and MPV.

Currently, physicians tend to focus on the relationship between the change of platelet count and diseases, while ignoring the significance of other platelet parameters in the diagnosis and treatment in diseases. The findings of the current study have demonstrated that the test of platelet parameters is convenient and reliable, and valuable to the diagnosis and follow up in ITP children as it avoids the pain from bone marrow biopsy, repeated tests, and good patient compliance and dynamic assessment of the recovery in ITP children is possible. Findings of the present study indicated that a good platelet count is important for proper assessment of platelet parameters (47). However, IPF is not affected by platelet count, thus it is complementary to platelet parameters in the etiology of thrombocytopenia and the evaluation of efficacy.

\section{Acknowledgements}

The present study was funded by the Sichuan Department of Education Scientific and Development Funds (2011ZA150).

\section{References}

1. Min $Z$ and Lin $X$ : The advancement in the treatment of the children with refractory idiopathic thrombocytopenic purpura. J Clin Pediatr 26: 727-730, 2008.

2. Terrell DR, Beebe LA, Vesely SK, Neas BR, Segal JB and George JN: The incidence of immune thrombocytopenic purpura in children and adults: a critical review of published reports. Am J Hematol 85: 174-180, 2010.

3. Hu Q: Diagnosis of idiopathic thrombocytopenic purpura in children. Journal of Applied Clinical Pediatrics 25: 159-161, 2010 (In Chinese).

4. Li P, Shi T, Gao J, Zhao D and Xiao A: The immunization in the children with acute immune thrombocytopenia. Chinese Journal of Practical Pediatrics 29: 548-550, 2014 (In Chinese).

5. Provan D and Newland AC: Newl and current management of primary immune thrombocytopenia. Adv Ther 32: 875-887, 2015.

6. Liu $W$ and Qian X: Interpretation of the recommendations for the diagnosis and treatment of pediatric primary immune thrombocytopenia. Chin J Obstet Gynecol Pediatr 21: 245-248, 2015.

7. Winkler J, Kroiss S, Rand ML, Azzouzi I, Annie Bang KW, Speer $\mathrm{O}$ and Schmugge M: Platelet apoptosis in paediatric immune thrombocy topenia is ameliorated by intravenous immunoglobulin. Br J Haematol 156: 508-515, 2012.

8. Yin J, Wang J, Chen XQ, Yu WJ, Liao JY and Wang DX: Investigation on platelet apoptosis in patients with chronic idiopathic thrombocytopenic purpura. J Diagn Concepts \& Pract 9: 236-241, 2010 (In Chinese).

9. Korkmaz S, Uslu AU, Aydin B, Dogan O and Sencan M: Pre-treatment and post-treatment changes in platelet indices in patients with immune thrombocytopenia. Saudi Med J 34: 591-596, 2013.

10. Ntaios G, Papadopoulos A, Chatzinikolaou A, Saouli Z, Karalazou P, Kaiafa G, Girtovitis F, Kontoninas Z, Savopoulos C, Hatzitolios A, et al: Increased values of mean platelet volume and platelet size deviation width may provide a safe positive diagnosis of idiopathic thrombocytopenic purpura. Acta Haematol 119: 173-177, 2008.

11. Chen JF, Yang LH, Chang LX, Feng JJ and Liu JQ: The clinical significance of circulating $\mathrm{B}$ cells secreting anti-glycoprotein IIb/IIIa antibody and platelet glycoprotein IIb/IIIa in patients with primary immune thrombocytopenia. Hematology 17 : 283-290, 2012.

12. Thomas MR, Wijeyeratne YD, May JA, Johnson A, Heptinstall S and Fox SC: A platelet P-selectin test predicts adverse cardiovascular events in patients with acute coronary syndromes treated with aspirin and clopidogrel. Platelets 25 : 612-618, 2014.

13. He Y, Zhao YX, Zhu MQ, Wu Q and Ruan CG: Detection of autoantibodies against platelet glycoproteins in patients with immune thrombocytopenic purpura by flow cytometric immunobead array. Clin Chim Acta 415: 176-180, 2013.

14. Xun L, Zhu WB, Wu JS, Cai XY, Liu X, Han YS, Yang HZ, Zheng CC and Li Q: Diagnosing ITP by Measuring the CD 61 and PAIg with FCM. Chin J Thromb Hemost 17: 8-12, 2011 (In Chinese).

15. Wang T, Zhang $\mathrm{C}$ and Lu J: Platelet parameter and platelet membrane glycoprotein in childhood acute lymphoblastic leukemia. J Chin Pediatr Blood Cancer 11: 12-16, 2006 (In Chinese). 
16. Psaila B, Bussel JB, Frelinger AL, Babula B, Linden MD, Li Y, Barnard MR, Tate C, Feldman EJ and Michelson AD: Differences in platelet function in patients with acute myeloid leukemia and myelodysplasia compared to equally thrombocytopenic patients with immune thrombocytopenia. J Thromb Haemost 9: 2302-2310, 2011.

17. Subspecialty Group of Hematology, The Society of Pediatrics, Chinese Medical Association; Editorial Board, Chinese Journal of Pediatrics: Recommendations for diagnosis and treatment of primary immune thrombocytopenia in children. Zhonghua $\mathrm{Er}$ Ke Za Zhi 51: 382-384, 2013 (In Chinese).

18. Xu Q and Liu WJ: Advances in the diagnosis and treatment of primary immune thrombocytopenia in children. Chin J Pract Pediatr 28: 646-652, 2013 (In Chinese).

19. Huang Z, Liu WJ, Guo QL and Liu CY: Platelet parameters and expression of platelet membrane glycoprotein in childhood acute lymphoblastic leukemia. Genet Mol Res 14: 16074-16089, 2015

20. Sarratt KL, Chen H, Zutter MM, Santoro SA, Hammer DA and Kahn ML: GPVI and alpha2betal play independent critical roles during platelet adhesion and aggregate formation to collagen under flow. Blood 106: 1268-1277, 2005.

21. Huang Z and Liu W: Progress in research on platelet glycoproteins of thrombocytopenia in children. J Appl Clin Pediatr 29: 227-230, 2014 (In Chinese).

22. Mutlu A, Gyulkhandanyan AV, Freedman J and Leytin V: Concurrent and separate inside-out transition of platelet apoptosis and activation markers to the platelet surface. Br J Haematol 163 377-384, 2013

23. Serebruany V, Malinin A, Aradi D, Kuliczkowski W, Norgard NB and Boden WE: The in vitro effects of niacin on platelet biomarkers in human volunteers. Thromb Haemost 104: 311-317, 2010.

24. Cines DB and Blanchette VS: Immune thrombocytopenic purpura. N Engl J Med 346: 995-1008, 2002.

25. Dong NZ, Cui RJ and Ruan CG: Generation of human Fab antibody against platelet membrane glycoprotein IIb/IIIa and its effect on platelet aggregation. Chin J Cell Mol Immunol 25 65-67, 2009 (In Chinese)

26. Yang G: research and application of platelet glycoprotein iib/iiia acceptor antagonist. Clin Med Eng 17: 149-151, 2010.

27. Kashiwagi $\mathrm{H}$ and Tomiyama $\mathrm{Y}$ : Pathophysiology and management of primary immune thrombocytopenia. Int J Hematol 98: 24-33, 2013.

28. Bhoria P, Sharma S, Varma N, Malhotra P, Varma S and Luthra-Guptasarma M: Effect of steroids on the activation status of platelets in patients with Immune thrombocytopenia (ITP) Platelets 26: 119-126, 2015.

29. Wang C, Niu A, Lu Z, Yang X, Wang L and Zou X: The clinical significances of PAIgG, PAC-1 and CD62P in patients with idiopathic thrombocytopenic purpura. Zhejiang J Lab Med 3: 7-10, 2005 (In Chinese).

30. Psaila B, Bussel JB, Linden MD, Babula B, Li Y, Barnard MR, Tate C, Mathur K, Frelinger AL and Michelson AD: In vivo effects of eltrombopag on platelet function in immune thrombocytopenia: no evidence of platelet activation. Blood 119: 4066-4072, 2012.

31. Vinholt PJ, Hvas AM and Nybo M: An overview of platelet indices and methods for evaluating platelet function in thrombocytopenic patients. Eur J Haematol 92: 367-376, 2014.

32. Zhang Y and Gang W: Study on clinical significance of platelet relevant parameters in diagnosis of thrombocytopenia. J Clin Exp Med 12: 1533-1535, 2013.
33. Bai J and Liu W: Changes of platelet parameters and platelet function in primary immune thrombocytopenia. Chin J Pract Pediatr 30: 150-153, 2015 (In Chinese).

34. Adly AA, Ragab IA, Ismail EA and Farahat MM: Evaluation of the immature platelet fraction in the diagnosis and prognosis of childhood immune thrombocytopenia. Platelets 26: 645-650, 2015.

35. Balduini CL and Noris P: Mean platelet volume for distinguishing between inherited thrombocytopenias and immune thrombocytopenia - response to Beyan. Br J Haematol 163: 413-414, 2013

36. Fan $\mathrm{L}$ and Wei W: Platelet parameters in children with idiopathic thrombocytopenic purpura clinical application analysis. J Clin Exp Med 13: 124-126, 2014.

37. Strauss G, Vollert C, von Stackelberg A, Weimann A, Gaedicke G and Schulze H: Immature platelet count: A simple parameter for distinguishing thrombocytopenia in pediatric acute lymphocytic leukemia from immune thrombocytopenia. Pediatr Blood Cancer 57: 641-647, 2011.

38. Pons I, Monteagudo M, Lucchetti G, Muñoz L, Perea G, Colomina I, Guiu J and Obiols J: Correlation between immature platelet fraction and reticulated platelets. Usefulness in the etiology diagnosis of thrombocytopenia. Eur J Haematol 85: $158-163,2010$.

39. Jiang $\mathrm{W}$, Jiang $\mathrm{H}, \mathrm{Wu} \mathrm{Y}$, Zhang $\mathrm{Z}$ and Chen $\mathrm{X}$ : The variation of immature platelet fraction in patients with thrombocytopenic diseases. Lab Med 28: 47-50, 2013

40. Yang J, Zhao Y, Wu W, Hua B, Zhu Z, Yang R and Wang S: Value of Reticulated Platelet Countsin Identifying Thrombocytopenia Aetiology. J Exp Hematol 18: 482-485, 2010 (In Chinese).

41. Michur H, Maślanka K, Szczepiński A and Mariańska B: Reticulated platelets as a marker of platelet recovery after allogeneic stem cell transplantation. Int J Lab Hematol 30: 519-525, 2008.

42. Liu Y, Wang Z, Yuan B, Wang X, Wu Q and Yuan H: Role of reticulated platelets and platelet-associated antibody in differential diagnosis of idiopathic thrombocytopenic purpura. J Exp Hematol 19: 979-982, 2011 (In Chinese).

43. Xiao M, Liu J, Wu P and Yuan Q: Significance of platelet parameters measurement in thrombocytopenia disease. Int J Lab Med 34: 418-420, 2013 (In Chinese).

44. Adly AA, Ragab IA, Ismail EA and Farahat MM: Evaluation of the immature platelet fraction in the diagnosis and prognosis of childhood immune thrombocytopenia. Platelets 26: 645-650, 2015.

45. Barsam SJ, Psaila B, Forestier M, Page LK, Sloane PA, Geyer JT, Villarica GO, Ruisi MM, Gernsheimer TB, Beer JH, et al: Platelet production and platelet destruction: assessing mechanisms of treatment effect in immune thrombocytopenia. Blood 117: 5723-5732, 2011

46. Greene LA, Chen S, Seery C, Imahiyerobo AM and Bussel JB: Beyond the platelet count: Immature platelet fraction and thromboelastometry correlate with bleeding in patients with immune thrombocytopenia. Br J Haematol 166: 592-600, 2014.

47. Banfi G and Germagnoli L: Preanalytical phase in haematology. J Med Biochem 27: 348-353, 2008. 